\title{
Stratosphere-Troposphere Coupling in a Relatively Simple AGCM: Impact of the Seasonal Cycle
}

\author{
PAUL J. KuSHNER \\ Department of Physics, University of Toronto, Toronto, Ontario, Canada \\ LORENZO M. POLVANI \\ Department of Applied Physics and Applied Mathematics, and Department of Earth and Environmental Sciences, \\ Columbia University, New York, New York
}

(Manuscript received 2 December 2004, in final form 1 July 2005)

\begin{abstract}
The seasonal time dependence of the tropospheric circulation response to polar stratospheric cooling in a simple atmospheric general circulation model is investigated. When the model is run without a seasonal cycle, polar stratospheric cooling induces a positive annular-mode response in the troposphere that takes a remarkably long time-several hundred days - to fully equilibrate. One is thus led to ask whether the tropospheric response would survive in the presence of a seasonal cycle. When a seasonal cycle is introduced into the model stratosphere, the tropospheric response appears with a distinct time lag with respect to the stratospheric cooling, but, in the long-term mean, the pattern of the wind response is very similar to the one that results from stratospheric forcing in the absence of a seasonal cycle.
\end{abstract}

\section{Introduction}

Recent observational studies (e.g., Thompson and Solomon 2002, hereafter TS02) and comprehensive climate modeling studies (e.g., Sexton 2001; Gillett et al. 2002; Gillett and Thompson 2003, hereafter GT03) have suggested that polar-stratospheric cooling associated with photochemical ozone loss might influence the extratropical tropospheric circulation. Studies using relatively simple atmospheric GCMs (e.g., Polvani and Kushner 2002; Taguchi and Yoden 2002; Kushner and Polvani 2004; Song and Robinson 2004) have provided dynamical insights into this kind of downward influence.

In Polvani and Kushner (2002, hereafter PK02) and Kushner and Polvani (2004, hereafter KP04), we used a simplified AGCM to analyze the impact of externally imposed stratospheric cooling on the tropospheric circulation. In these studies, we found that, as the polar-

Corresponding author address: Dr. Paul J. Kushner, Dept. of Physics, University of Toronto, 60 St. George St., Toronto, ON, M5S 1A7, Canada.

E-mail: paul.kushner@utoronto.ca winter stratosphere is cooled, the tropospheric jet shifts poleward in a manner that projects almost entirely and positively (by convention) onto the model's annular mode. This result provides a simple qualitative representation of observed tropospheric circulation trends in the Southern Hemisphere that appear to be, at least in part, the result of ozone depletion (TS02; GT03). We find that the response is robust to variations in the strength of the reference polar vortex, in horizontal and vertical resolution, and in the strength of the spongelayer damping (PK02; KP04).

However, one unexplored simplifying assumption of our earlier studies might call into question the robustness of the tropospheric response. In PK02, to focus first on the simplest case and to improve statistical sampling, we used perpetual-solstice (i.e., time-independent) forcing. To examine the transient adjustment to equilibrium of the response, in KP04 we performed additional simulations in which the polar-stratospheric cooling is switched-on and thereafter held steady in time. We found that, whereas the stratosphere fully adjusts to the stratospheric cooling within a few radiative time scales, the troposphere fully adjusts to the stratospheric cooling over the course of several hundred days 

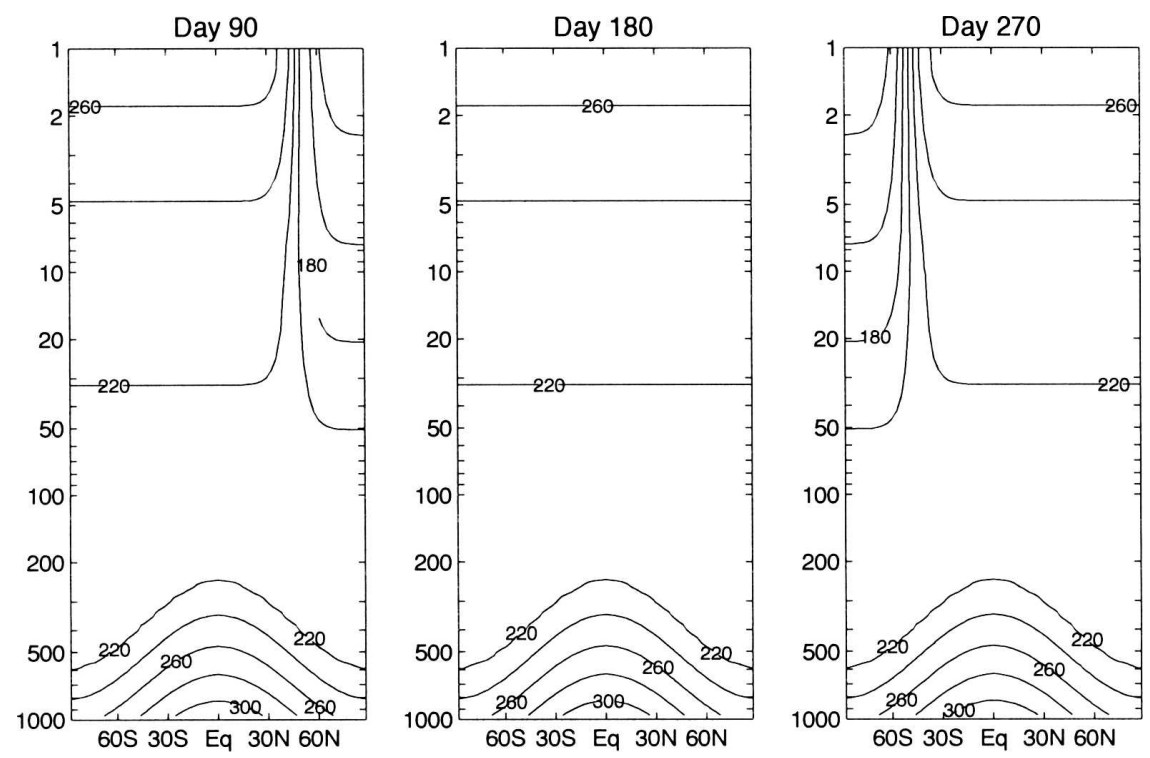

FIG. 1. Snapshots of $T_{\text {eq }}$ for SH (left) summer solstice, (middle) autumnal equinox, and (right) winter solstice. The contour interval is $20 \mathrm{~K}$.

(see Figs. 5 and 6 of KP04 and Fig. 2 in this study). This is an example of the well-known low-frequency variability and persistence that is present in this class of simple AGCM (e.g., James and James 1989; KP04; Reichler et al. 2005) and that may very well be unrealistic. The crux of the problem, therefore, is that these long time adjustment scales might prevent the troposphere from adjusting to the stratospheric cooling if a realistic seasonal cycle were added to the model. This might be the case, if, for example, the troposphere is sensitive to the peak strength of the winter polar vortex, but the influence of the polar vortex takes longer than the winter season to be felt in the troposphere.

In this note, we start from the viewpoint that it is worthwhile to better understand the characteristics of stratosphere-troposphere coupling in the PK02 model. We therefore leave aside the question of the realism of the model's low-frequency variability and focus instead on the question of whether the tropospheric response is robust to seasonal variations. We introduce a simple seasonal cycle formulation in the model stratosphere (section 2), examine the tropospheric response and its seasonal dependence in this case, and show that the response is indeed robust and related in a natural way to the perpetual-solstice forcing case of PK02 (section 3).

\section{Model}

Our model (see PK02; KP04; Reichler et al. 2005) is a dry, hydrostatic global primitive equations model with T42 resolution in the horizontal, 40-level resolution in the vertical, linear damping of the horizontal winds in a planetary boundary layer and in a sponge above $0.5 \mathrm{mb}$, a flat (i.e., spherical) lower boundary, and Newtonian relaxation toward a zonally symmetric equilibrium-temperature field $T_{\text {eq }}$. Except where noted below, the tropospheric part of $T_{\text {eq }}$, which we denote $T_{\text {eq }}^{\text {trop }}$, follows the Held and Suarez (1994) prescription. The stratospheric part of $T_{\text {eq }}$, which we denote $T_{\text {eq }}^{\text {strat }}$, blends polar night conditions in the winter hemisphere and standard atmosphere conditions in the summer hemisphere. A single parameter, $\gamma$, that is proportional to the vertical lapse rate of $T_{\text {eq }}$ at the poles, controls the equator-to-pole meridional temperature gradient in the winter hemisphere and hence the strength of the polar vortex.

For this study, we carry out simulations that use seasonal cycle and perpetual-solstice forms of $T_{\mathrm{eq}}^{\text {strat }}$. The seasonal cycle form of $T_{\mathrm{eq}}^{\text {strat }}$ is

$$
T_{\mathrm{eq}}^{\mathrm{strat}}(\phi, p, t)=[1-W(\phi, t)] T_{\mathrm{US}}(p)+W(\phi, t) T_{\mathrm{PV}}(p),
$$

where $\phi$ is latitude, $p$ is pressure, $T_{\mathrm{US}}(p)$ is the U.S. standard temperature (as in PK02) expressed as a function of pressure, and $T_{\mathrm{PV}}(p)$ is the polar vortex $T_{\mathrm{eq}}$ prescription of PK02. (The lapse rate of $T_{\mathrm{PV}}(p)$ is $\gamma$ in $\mathrm{K} / \mathrm{km}$.) The weighting function is

$$
\begin{aligned}
W(\phi, t)= & \frac{1}{2}\left\langle A_{S}(t)\left\{1+\tanh \left[\left(\phi-\phi_{0 S}\right) / \delta \phi_{S}\right]\right\}\right. \\
& \left.+A_{N}(t)\left\{1+\tanh \left[\left(\phi-\phi_{0 N}\right) / \delta \phi_{N}\right]\right\}\right\rangle,
\end{aligned}
$$





FIG. 2. The zonal-mean zonal wind response to switch-on forcing, as described in the text. The panel labeled "Month 1" represents the time mean over the first 30 days; "Month 2," the next 30 days, etc. The final panel represents the long time difference (a 9000-day mean) for the perpetual-solstice $\gamma=2$ and $\gamma=4$ integrations of PK02. The dashed contours are negative and the contours plotted are $\pm 2,4,6,12,18,24, \ldots \mathrm{m} \mathrm{s}^{-1}$. The shading indicates a $6 \mathrm{~m} \mathrm{~s}^{-1}$ contour interval.

where $A_{S}(t)=\max \left\{0.0, \sin \left[2 \pi\left(t-t_{0}\right) / \Delta T\right]\right\}, t_{0}=180 \mathrm{~d}$, $\Delta T=360$ days, $A_{N}(t)=\max [0.0, \sin (2 \pi t / 360)], \phi_{0 S}=$ $-50^{\circ}, \delta \phi_{\mathrm{S}}=-10^{\circ}, \phi_{0 N}=50^{\circ}$, and $\delta \phi_{N}=10^{\circ}$ latitude. At a given polar latitude, $T_{\text {eq }}^{\text {strat }}(\phi, p, t)$ thus varies between polar winter and polar summer over a 360-day year. The perpetual-solstice form of $T_{\text {eq }}^{\text {strat }}$ corresponds to $A_{S}(t)=1, A_{N}=0$, so that the weighting function $W$ corresponds to perpetual-solstice conditions in the Southern Hemisphere (SH); this form is as in PK02.
Figure 1 illustrates the seasonal cycle of $T_{\text {eq }}$ for the $\gamma$ $=4$ case for $\mathrm{SH}$ summer solstice, autumnal equinox, and winter solstice. Notice that the tropospheric portion of $T_{\text {eq }}$ (i.e., $T_{\text {eq }}^{\text {trop }}$ ) is time independent and symmetric upon reflection in latitude about the equator. In PK02 and KP04, on the other hand, $T_{\text {eq }}^{\text {trop }}$ is weakly asymmetric in latitude about the equator: the winterhemisphere baroclinicity is slightly increased by setting $\varepsilon=0.1$ in PK02 Eq. (A4). We set $\varepsilon=0$ in this study, 


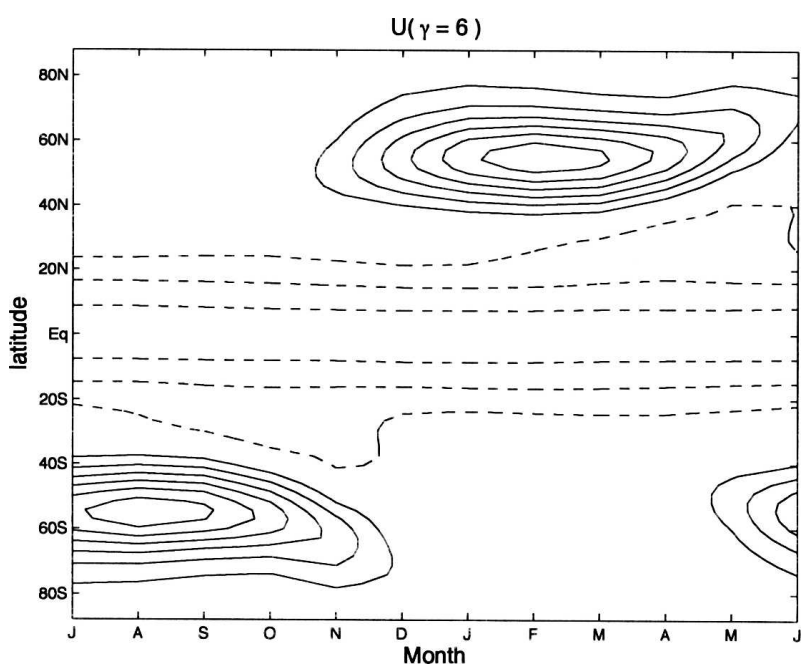

FIG. 3. Seasonal cycle of $10-\mathrm{hPa}$ zonal-mean zonal winds for the $\gamma=6$ case. The dashed contours are negative, the contours start from $\pm 10 \mathrm{~m} \mathrm{~s}^{-1}$, and the contour interval is $10 \mathrm{~m} \mathrm{~s}^{-1}$.

which implies that the seasonal-cycle $T_{\mathrm{eq}}$ is symmetric upon reflection in latitude about the equator and shifting in time by 180 days. This simplifies the forcing and improves sampling, because a similar symmetry applies to the long-term statistics of the simulations.

\section{Results}

For clarity, we start by recalling the model response to switch-on cooling in the stratosphere (KP04). This case does not have a seasonal cycle; it consists of 10 independent realizations in which the value of $\gamma$ has been switched from 2 to 4 at time zero and thereafter held steady. Figure 2 shows the ensemble mean of the zonal-mean zonal wind for these cases, minus the zonalmean zonal wind for the long-term mean of a timeindependent $\gamma=2$ simulation (the data are from the KP04 integrations; see that paper for further details). Notice that the contour interval is not uniform in the figure. The first 11 panels plot the time mean response over successive 30-day periods (labeled "Month 1," "Month 2," etc. in the figure); the last panel plots the long-term response. Over the first 3 to 4 months, the stratospheric winds increase in strength: this is the direct response to the stratospheric cooling. As the stratospheric response intensifies, a tropospheric wind response develops that becomes strongly established by month 8 but is not fully equilibrated even after month 11. The dipole pattern of the tropospheric response represents a positive annular mode pattern: this is the indirect response to the stratospheric cooling (PK02).

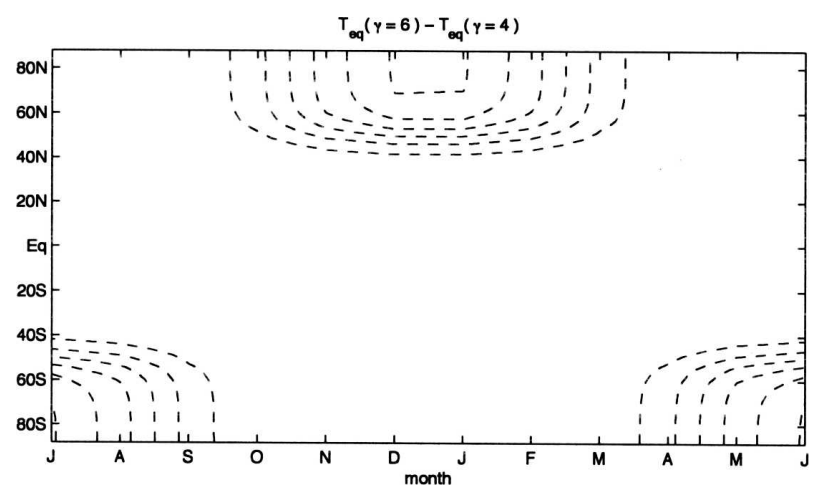

FIG. 4. Seasonal cycle of $\delta T_{\mathrm{eq}}^{\mathrm{strat}}(t)$ plotted as a function of calendar month and latitude. Contour interval is $5 \mathrm{~K}$ and the dashed contours denote negative values.

Because it takes several months for the signal in Fig. 2 to descend into the troposphere and become fully established, we now wish to determine whether the introduction of a seasonal cycle in $T_{\text {eq }}^{\text {strat }}$ would attenuate the stratospheric circulation response and therefore attenuate or otherwise change the tropospheric circulation response. To answer this question, we perform three integrations with the seasonal-cycle $T_{\mathrm{eq}}^{\text {strat }}$ given by Eqs. (1) and (2), for $\gamma=2, \gamma=4$, and $\gamma=6$. Figure 3 shows the seasonal cycle of the zonal-mean zonal wind at $10 \mathrm{hPa}$ for the $\gamma=6$ case. This represents a climatology from a 30-yr analysis period after a 3-yr spinup. ${ }^{1}$ The peak strength of the $10-\mathrm{hPa}$ westerlies lies between observed Northern Hemisphere (NH) and SH values (see, e.g., Randel 1992, p. 167). As with other aspects of the tropical stratospheric circulation in this model, the strong tropical easterlies are somewhat unrealistic and sensitive to variations of model parameters. As in PK02 and KP04, we now focus upon the extratropical circulation.

Because the stratospheric polar-winter conditions are only present for part of the year in the seasonal-cycle case, for a given value of the polar-winter lapse rate $\gamma$, the stratospheric zonal winds are weaker in the seasonal-cycle case than in the perpetual-solstice case. For example, for $\gamma=4$, the peak zonal winds at $10 \mathrm{mb}$ are about $40 \mathrm{~m} \mathrm{~s}^{-1}$ in the seasonal-cycle case, whereas the long-term mean zonal winds are about $90 \mathrm{~m} \mathrm{~s}^{-1}$ in the perpetual-solstice case (KP04). For $\gamma=2$, the strato-

\footnotetext{
${ }^{1}$ We plot monthly means in the figure. October corresponds to days 0-29 in Eq. (2), November to days 30-59, and so on. Thus, the Northern Hemisphere winter solstice (day 90) corresponds to the first day of January. The results we present here are similar for a 15 -yr analysis period.
} 



FIG. 5. The long-term mean difference of zonal-mean zonal wind between the $\gamma=4$ and $\gamma=6$ seasonal cycle cases. The dashed contours are negative and the contours plotted are \pm 1 , $2,3,6,9,12,15, \ldots \mathrm{m} \mathrm{s}^{-1}$. The shading indicates a $3 \mathrm{~m} \mathrm{~s}^{-1}$ contour interval.

spheric winds become unrealistically weak. Given this, we focus on the difference between the $\gamma=4$ and $\gamma=$ 6 seasonal-cycle cases, which best illustrates the key result we are presenting here.

The difference between the $\gamma=4$ and $\gamma=6$ seasonalcycle cases is the response to a stratospheric cooling written schematically as

$$
\delta T_{\text {eq }}^{\text {strat }}(t)=T_{\text {eq }}^{\text {strat }}(\gamma=6 ; t)-T_{\text {eq }}^{\text {strat }}(\gamma=4 ; t) .
$$

The quantity $\delta T_{\text {eq }}^{\text {strat }}$ is shown at $10 \mathrm{hPa}$ as a function of calendar month in Fig. 4; notice that by construction the cooling perturbation peaks during winter solstice. Cross sections of the zonal-wind response to $\delta T_{\mathrm{eq}}^{\text {strat }}(t)$, that is, of the long-term mean difference between the $\gamma=4$ and $\gamma=6$ integrations, are plotted for each climatological month in Fig. $5^{2}$.

Our main result is that the seasonal evolution in response to seasonally varying cooling in Fig. 5 is quali-

\footnotetext{
${ }^{2}$ Figure 5 combines $\mathrm{NH}$ and $\mathrm{SH}$ data using the symmetry described at the end of section 2 .
} 



FIG. 6. The zonal-mean zonal wind response for (a) the long-term mean of the perpetualsolstice cases and (b) the long-term annual mean of the seasonal-cycle cases, multiplied by 5 . Panel (b) represents the combined average of the $\mathrm{NH}$ and $\mathrm{SH}$ data, with the $\mathrm{NH}$ data reflected about the equator; this takes advantage of the symmetry of the annual mean statistics about the equator. The dashed contours are negative and the contours plotted are $\pm 2,4,6,8,16$, $24, \ldots \mathrm{m} \mathrm{s}^{-1}$. The shading indicates an $8 \mathrm{~m} \mathrm{~s}^{-1}$ contour interval.

tatively similar to the transient evolution in response to switch-on cooling in Fig. 2. Both figures show a similar strengthening of the polar vortex followed by a positive annular-mode response in the troposphere with a lag consistent with the known long time scales in the model. This demonstrates that in the seasonal cycle case, polar stratospheric cooling can induce a tropospheric response in the winds that is similar to the perpetual-solstice case, even though the cooling is only present for part of the year. There are, of course, significant differences between the figures. Notice, for example, that the contour interval in Fig. 5 is half of that in Fig. 2: because the forcing is generally weaker in the seasonally varying case, so is the response. Furthermore, the tropospheric response in Fig. 5 persists for several months into summer, even after the stratospheric cooling has weakened, which is another signature of the long-time-scale persistence of the tropospheric annular mode in this model (Reichler et al. 2005). The response to changing $\gamma$ from 2 to 4 presents a similar seasonal evolution (figure not shown).

One final important issue is the degree to which the seasonal cycle affects the coupling between the stratosphere and the troposphere. We partially answer this question by carrying out perpetual-solstice simulations. We run $\gamma=2$ and $\gamma=4$ simulations with a 3500-day analysis period after a 1000-day spinup (the results for a 1500-day analysis period are similar). These correspond to the same cases as the ones plotted in Fig. 2, except that we have changed the hemispheric asymmetry parameter from the value $\varepsilon=0.1$ in KP04 to $\varepsilon=0.0$ in PK02. Figure 6a plots the zonal-mean zonal wind response for these cases. ${ }^{3}$ One measure of the strength of the stratosphere-troposphere coupling of the response is the ratio of the amplitude of the midtropospheric response to the stratospheric response. In the perpetual-solstice case, this is about 1:5. This ratio has a strong seasonal variation in Fig. 5, ranging from almost zero in July to 1:1 in December. The annual mean response, which is shown multiplied by a factor of 5 in Fig. 6b, averages over this behavior and shows that the ratio and the overall pattern of the response is quite robust in the two cases.

\section{Conclusions}

In PK02 and KP04, we suggested that at least some portion of the tropospheric sensitivity to stratospheric thermal perturbations might be attributed to the interaction between the zonal-mean flow and transient eddies on both the synoptic and planetary scales. However, given the long adjustment scales characteristic of models such as ours, this suggestion might be invalidated were a seasonal cycle able to wipe out the stratospheric influence on the troposphere. In this note, we have shown that the stratosphere-troposphere coupled

\footnotetext{
${ }^{3}$ Comparing Fig. 6a with the last panel in Fig. 2, we see that setting $\varepsilon=0$ for $T_{\mathrm{eq}}^{\text {trop }}$ has evidently weakened the tropospheric response relative to the stratospheric response in this case. We have no simple explanation for this sensitivity to $\varepsilon$.
} 
responses to stratospheric cooling in a relatively simple model such as ours are similar for the seasonal cycle and perpetual-solstice cases. This increases our confidence that the transient eddy mean-flow interactions are important to understanding the coupled stratosphere-troposphere system, and in the robustness of the model's qualitative behavior. The magnitude of the response is relatively weaker in the seasonal cycle case because there is not enough time for the response to fully adjust. But both cases exhibit a positive annularmode response in the troposphere, and the degree of stratosphere-troposphere coupling is similar in the two cases.

This analysis still leaves the issue of the relatively slow time scales of stratosphere-troposphere coupling unaddressed. We attribute this to the relatively weak eddy forcing of the stratosphere by the troposphere, and to the long-range tropospheric persistence in the model (KP04; Reichler et al. 2005; I. Held 2005, personal communication; E. Gerber 2005, personal communication). Both these characteristics might be sensitive to the presence of topography and other changes to the model formulation, which is a direction we are currently exploring.

Acknowledgments. This work was completed while P. J. Kushner was attending the Aspen Center for Physics program on Novel Approaches to Climate. P. J. Kushner acknowledges the support of the Natural Sciences and Engineering Research Council of Canada. The work of L. M. Polvani is supported, in part, by a research award from the National Science Foundation.

\section{REFERENCES}

Gillett, N. P., and D. Thompson, 2003: Simulation of recent Southern Hemisphere climate change. Science, 302, 273-275.

_, M. R. Allen, and K. D. Williams, 2002: The role of stratospheric resolution in simulating the Arctic Oscillation response to greenhouse gases. Geophys. Res. Lett., 29, 1500, doi:10.1029/2001GL014444.

Held, I. M., and M. J. Suarez, 1994: A proposal for the intercomparison of the dynamical cores of atmospheric general circulation models. Bull. Amer. Meteor. Soc., 75, 1825-1830.

James, I. N., and P. James, 1989: Ultra-low-frequency variability in a simple atmospheric circulation model. Nature, 342, 5355.

Kushner, P., and L. Polvani, 2004: Stratosphere-troposphere coupling in a relatively simple AGCM: The role of eddies. $J$. Climate, 17, 629-639.

Polvani, L. M., and P. Kushner, 2002: Tropospheric response to stratospheric perturbations in a relatively simple general circulation model. Geophys. Res. Lett., 29, 1114, doi:10.1029/ 2001 GL014284.

Randel, W. J., 1992: Global atmospheric circulation statistics, 1000-1 mb. NCAR Tech. Note NCAR/TN-366+STR.

Reichler, T. J., P. J. Kushner, and L. M. Polvani, 2005: The coupled stratosphere troposphere response to impulsive forcing from the troposphere. J. Atmos. Sci., 62, 3337-3352.

Sexton, D. M. H., 2001: The effect of stratospheric ozone depletion on the phase of the Antarctic Oscillation. Geophys. Res. Lett., 28, 3697-3700.

Song, Y., and W. A. Robinson, 2004: Dynamical mechanisms for stratospheric influences on the troposphere. J. Atmos. Sci., 61, 1711-1725.

Taguchi, M., and S. Yoden, 2002: Internal interannual variations of the troposphere-stratosphere coupled system in a simple global circulation model. Part I: Parameter sweep experiment. J. Atmos. Sci., 59, 3021-3036.

Thompson, D. W. J., and S. Solomon, 2002: Interpretation of recent Southern Hemisphere climate change. Science, 296, 895-899. 